\title{
ON OPIAL-TYPE INTEGRAL INEQUALITIES AND APPLICATIONS
}

\author{
CHANG-JiAn ZHAO AND WING-SUM CHEUNG
}

Abstract. In the present paper we establish some new Opial-type inequalities involving higher order partial derivatives. Our results in special cases yield some of the recent results on Opial's inequality. As application, we prove the uniqueness of the solution of initial value problem involving higher order partial differential equation.

Mathematics subject classification (2010): 26D15.

Keywords and phrases: Opial's integral inequality, Cauchy-Schwarz inequality, Hölder's inequality.

\section{REFERENCES}

[1] R. P. Agarwal and P. Y. H. PAng, Opial Inequalities with Applications in Differential and Difference Equations, Kluwer Academic Publishers, Dordrecht, 1995.

[2] R. P. Agarwal and V. LaKshmikantham, Uniqueness and Nonuniqueness Criteria for Ordinary Differential Equations, World Scientific, Singapore, 1993.

[3] R. P. Agarwal and E. Thandapani, On some new integrodifferential inequalities, Anal. sti. Univ. "Al. I. Cuza" din Iasi 28 (1982), 123-126.

[4] D. Bainov And P. Simeonov, Integral Inequalities and Applications, Kluwer Academic Publishers, Dordrecht, 1992.

[5] P. R. BeESACK, On an integral inequality of Z. Opial, Trans. Amer. Math. Soc. 104 (1962), 470-475.

[6] W. S. Cheung, On Opial-type inequalities in two variables, Aequationes Math. 38 (1989), 236-244.

[7] W. S. Cheung, Some new Opial-type inequalities, Mathematika 37 (1990), 136-142.

[8] W. S. Cheung, Some generalized Opial-type inequalities, J. Math. Anal. Appl. 162 (1991), 317-321.

[9] W. S. Cheung, Opial-type inequalities with $m$ functions in $n$ variables, Mathematika 39 (1992), 319-326.

[10] W. S. Cheung, D. D. Zhao And J. E. PeČARIĆ, Opial-type inequalities for Differential Operators, to appear in: Nonlinear Anal.

[11] K. M. DAS, An inequality similar to Opial's inequality, Proc. Amer. Math. Soc. 22 (1969), 258-261.

[12] E. K, Godunova AND V. I. Levin, On an inequality of Maroni, Mat. Zametki 2 (1967), 221-224.

[13] HuA L. K., On an inequality of Opial, Scientia Sinica 14 (1965), 789-790.

[14] J. D. LI, Opial-type integral inequalities involving several higher order derivatives, J. Math. Anal. Appl. 167 (1992), 98-100.

[15] D. S. Mitrinovič, J. E. PeČArić And A. M. Fink, Inequalities involving Functions and Their Integrals ang Derivatives, Kluwer Academic Publishers, Dordrecht, 1991.

[16] D. S. Mitrinovič, Analtic Inequalities, Springer-Verlag, Berlin, New York, 1970.

[17] Z. Opial, Sur une inégalité, Ann. Polon. Math. 8 (1960), 29-32.

[18] B. G. Pachpatte, On some new generalizations of Opial inequality, Demonstratio Math. 19 (1986), 281-291.

[19] B. G. PachPatte, On Opial-type integral inequalities , J. Math. Anal. Appl. 120 (1986), 547-556.

[20] B. G. Pachpatte, Some inequalities similar to Opial's inequality, Demonstratio Math. 26 (1993), 643-647.

[21] B. G. Pachpatte, A note on generalized Opial type inequalities, Tamkang J. Math. 24 (1993), 229_ 235.

[22] J. E. PeČARIĆ, An integral inequality, in Analysis, Geometry, and Groups: A Riemann Legacy Volume (H. M. Srivastava and Th. M. Rassias, Editors), Part II, Hadronic Press, Palm Harbor, Florida, 1993, pp. $472-478$. 
[23] J. E. PeČARIĆ AND I. BRnetić, Note on generalization of Godunova-Levin-Opial inequality, Demonstratio Math. 30 (1997), 545-549.

[24] J. E. PEČARIĆ AND I. BRnETIĆ, Note on the Generalization of Godunova-Levin-Opial inequality in Several Independent Variables, J. Math. Anal. Appl. 215 (1997), 274-282.

[25] G. I. Rozanova, Integral inequalities with derivatives and with arbitrary convex functions, Moskov. Gos. Ped. Inst. Vcen. Zap. 460 (1972), 58-65.

[26] G. S. YAng, Inequality of Opial-type in two variables, Tamkung J. Math. 13 (1982), 255-259. 